\title{
Fecal calprotectin in daily practice for patients with inflammatory bowel disease: where do we stand in Korea?
}

Jae Myung Cha

Department of Internal Medicine, Kyung Hee University School of Medicine, Seoul, Korea

Received: November 7, 2018 Accepted: November 18, 2018

\section{Correspondence to}

Jae Myung Cha, M.D.

Department of Internal

Medicine, Kyung Hee

University Hospital at

Gangdong, 892 Dongnam-ro,

Gangdong-gu, Seoul 05278,

Korea

Tel: +82-2-440-6113

Fax: +82-2-440-7849

E-mail: drcha@khu.ac.kr

\section{See Article on Page 72-80}

Inflammatory bowel disease (IBD) is characterized by recurrent episodes of inflammation in the intestinal tract. Diagnosis and assessment of IBD are based on patient symptoms, serological tests, radiology, and endoscopy. However, the ability to monitor inflammation and predict disease progression in individual patients with IBD is limited. Patient symptoms are an important indicator of inflammation and disease activity in IBD, but are subjective and potentially influenced by other non-inflammatory features of IBD [1]. Serological tests are widely used to assess the disease activity of IBD, but they exhibit low sensitivity and specificity for diagnosing intestinal inflammation and poor correlations with symptoms and disease activity indices [2]. Radiology is useful for locating intestinal inflammation but is limited by its cost, suboptimal sensitivity and/or specificity, and exposure of the patient to ionizing radiation [1]. Endoscopy is the current gold standard for assessing intestinal inflammation but is somewhat invasive and limited by its cost and uncomfortable bowel preparation procedure. These limitations prevent frequent assessment of disease activity by endoscopic techniques.
Thus, fecal calprotectin (FC) has emerged as a new diagnostic tool to detect and monitor intestinal inflammation in IBD, as it is a simple, rapid, sensitive, specific, inexpensive, and non-invasive marker of inflammation [1]. Calprotectin, an abundant calcium-binding protein that belongs to the S1oo family, is derived predominantly from neutrophils and to a lesser extent monocytes and reactive macrophages [3]. FC is a sensitive marker of intestinal inflammation in IBD, as its concentration reflects the migration of neutrophils into the gut lumen. FC is measured by enzyme-linked immunosorbent assay (ELISA), with interassay and intra-assay coefficients of variation of $15 \%$ and $2 \%$, respectively [4]. A meta-analysis of the diagnostic accuracy of FC in IBD showed that this test was able to differentiate patients with IBD from those with non-organic diseases, with a pooled sensitivity and specificity of $93 \%$ and $96 \%$, respectively, at cut-off levels ranging from 24 to $150 \mu \mathrm{g} / \mathrm{g}[5,6]$. FC also has a good correlation with disease activity and may be a useful predictor of relapse or response to therapy in IBD. However, currently, FC is not widely used in clinical practice in Korea.

ELISA, which is the current method used to measure FC, has disadvantages in that it is a time-consuming pro- 
cess that requires expertise and multiple samples to be run at the same time. However, the recently developed quantitative point-of-care test (QPOCT) is simple, easy to use, and capable of producing more rapid results compared with ELISA. Unfortunately, studies comparing the two methods in terms of accuracy and availability are lacking [7]. In the latest issue of the Korean Journal of Internal Medicine, Lee et al. [8] reported the usefulness of FC as a marker of disease activity in IBD by examining the correlations between the FC level and laboratory and endoscopic parameters in patients with IBD; they also compared FC measurements between the ELISA and QPOCT methods. In that prospective study, fecal samples and clinical data were collected from consecutive patients with IBD, and FC levels were measured by both ELISA and QPOCT. According to the authors, there was a strong correlation between the FC level determined by ELISA and clinical activity indices $(p<0.05)$. The FC level was significantly lower in patients with mucosal healing compared with those without mucosal healing in ulcerative colitis $(p=0.005)$. The FC levels measured by ELISA and QPOCT showed very close correlations in both ulcerative colitis and Crohn's disease (both $p=0.000$ ). Cut-off levels of 201.3 and $150.5 \mu \mathrm{g} / \mathrm{g}$ for FC measured by ELISA and QPOCT, respectively, predicted endoscopic inflammation (Mayo endoscopic subscore $\geq 1$ ) in ulcerative colitis, with sensitivities of $81.8 \%$ and $85.8 \%$, respectively, and specificities of $100 \%$ for both cut-off levels [8]. These findings by Lee et al. [8] provide relevant information to clinicians in Korea and could be clinically meaningful despite the small number of patients with IBD from a single center in their study.

However, in interpreting the data from Lee et al. [8], it is important to appreciate the limitations of FC measurements. A study that compared six different assays for FC measurement in patients with IBD found a fivefold quantitative difference among the assays, highlighting the need for standardization [4,9]. Aside from the need for longitudinal measurements, there is considerable intra-individual variation in the FC level throughout the course of a day, and therefore therapeutic decisions should not be based on single measurements [10]. As discussed by Lee et al. [8], their findings were limited by the lack of FC follow-up data in patients with IBD. To demonstrate the close correlation between FC and disease activity, FC measurements taken at different time points from the same patient and in patients with varying degrees of disease activity may be more useful and informative. Therefore, large-scale studies involving patients with IBD of varying disease activities may be warranted to overcome this issue.

In summary, FC is a reliable surrogate marker of endoscopic activity in Korean patients with IBD. Therefore, FC measurement has the potential to replace colonoscopy for serial assessment of mucosal inflammation in IBD patients. Considering the excellent correlation between the FC level measured by ELISA and that by the rapid QPOCT, QPOCT appears to be more suitable for real practice and can be used more widely in the future, as it is easy to perform and generates more rapid quantitative results compared with ELISA.

\section{Conflict of interest}

No potential conflict of interest relevant to this article was reported.

\section{REFERENCES}

1. Konikoff MR, Denson LA. Role of fecal calprotectin as a biomarker of intestinal inflammation in inflammatory bowel disease. Inflamm Bowel Dis 2006;12:524-34.

2. Nielsen $\mathrm{OH}$, Vainer B, Madsen SM, Seidelin JB, Heegaard NH. Established and emerging biological activity markers of inflammatory bowel disease. Am J Gastroenterol 2000;95:359-367.

3. Bjerke K, Halstensen TS, Jahnsen F, Pulford K, Brandtzaeg P. Distribution of macrophages and granulocytes expressing L1 protein (calprotectin) in human Peyer's patches compared with normal ileal lamina propria and mesenteric lymph nodes. Gut 1993;34:1357-1363.

4. Roseth AG, Fagerhol MK, Aadland E, Schjonsby H. Assessment of the neutrophil dominating protein calprotectin in feces. A methodologic study. Scand J Gastroenterol 1992;27:793-798.

5. Menees SB, Powell C, Kurlander J, Goel A, Chey WD. A meta-analysis of the utility of C-reactive protein, erythrocyte sedimentation rate, fecal calprotectin, and fecal lactoferrin to exclude inflammatory bowel disease in adults with IBS. Am J Gastroenterol 2015;110:444-454.

6. Roseth AG, Aadland E, Grzyb K. Normalization of faecal calprotectin: a predictor of mucosal healing in patients 
with inflammatory bowel disease. Scand J Gastroenterol 2004;39:1017-1020.

7. Wassell J, Wallage M, Brewer E. Evaluation of the Quantum Blue rapid test for faecal calprotectin. Ann Clin Biochem 2012;49(Pt 1):55-58.

8. Lee YW, Lee KM, Lee JM, et al. The usefulness of fecal calprotectin in assessing inflammatory bowel disease activity. Korean J Intern Med 2019;34:72-8o.
9. Panes J, Jairath V, Levesque BG. Advances in use of endoscopy, radiology, and biomarkers to monitor inflammatory bowel diseases. Gastroenterology 2017;152:362-373.e3.

10. Calafat M, Cabre E, Manosa M, Lobaton T, Marin L, Domenech E. High within-day variability of fecal calprotectin levels in patients with active ulcerative colitis: what is the best timing for stool sampling? Inflamm Bowel Dis 2015;21:1072-1076. 